\title{
Miranda
}

Revue pluridisciplinaire du monde anglophone /

Multidisciplinary peer-reviewed journal on the English-

speaking world

$20 \mid 2020$

Staging American Nights

\section{Claudine Armand, dir., Voix et silence dans les arts : Passages, poïesis et performativité}

\section{Christelle Serée-Chaussinand}

\section{OpenEdition}

Journals

Édition électronique

URL : http://journals.openedition.org/miranda/23309

DOI : 10.4000/miranda.23309

ISSN : 2108-6559

Éditeur

Université Toulouse - Jean Jaurès

\section{Référence électronique}

Christelle Serée-Chaussinand, «Claudine Armand, dir., Voix et silence dans les arts : Passages, poïèsis et performativité », Miranda [En ligne], 20 | 2020, mis en ligne le 19 mars 2020, consulté le 16 février 2021 URL : http://journals.openedition.org/miranda/23309; DOI : https://doi.org/10.4000/miranda.23309

Ce document a été généré automatiquement le 16 février 2021.

\section{cc) (i) () $\Theta$}

Miranda is licensed under a Creative Commons Attribution-NonCommercial-NoDerivatives 4.0 International License. 
Claudine Armand, dir., Voix et silence dans les arts : Passages, poïèsis et performativité

Christelle Serée-Chaussinand

\section{RÉFÉRENCE}

Claudine Armand, dir., Voix et silence dans les arts : Passages, poïèsis et performativité

(Nancy, Éditions Universitaires de Lorraine, 2019), 251 pages, ISBN 978-2-8143-0531-1 


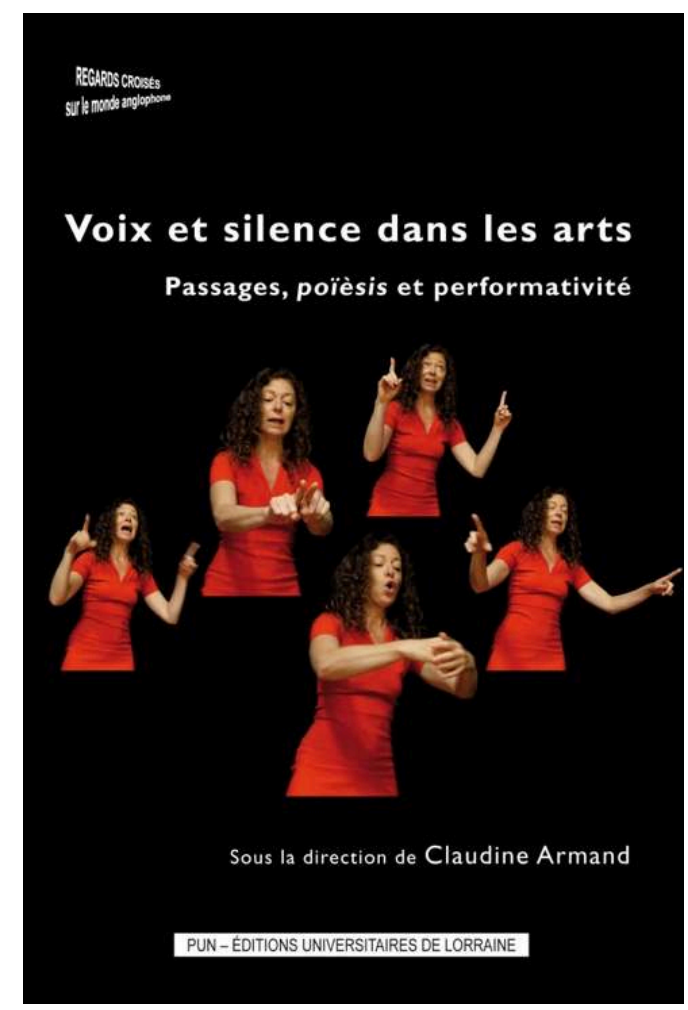

1 Citant Merleau-Ponty, Claudine Armand nous invite, dès les premières pages de cet ouvrage, à être « sensibles à ces fils de silence dont le tissu de la parole est entremêlé » et remarque que le silence troue la voix tout autant que la voix troue le silence (11). Quiconque a assisté à une représentation de Not I de Samuel Beckett ou simplement visionné un enregistrement de la performance de Billie Whitelaw qui a créé la pièce à Londres en 1973, ne peut qu'acquiescer. Dans ce monologue dramatique où l'écrivain irlandais pousse la théâtralité à l'extrême, une bouche flotte comme en apesanteur dans le noir opaque de la salle, déversant un torrent de mots. Mais ce qui marque, ce ne sont pas tant les mots proférés que cette voix féminine qui perce progressivement le silence, s'enfle et s'infléchit, file sur la crête incertaine d'un souffle qui s'étire jusqu'à l'asphyxie, puis est à nouveau happée par un silence assourdissant. Dans cette pièce singulière où le visuel réduit à une bouche béante est obscène, où le vocal l'emporte sur le verbal, Beckett rend perceptible que la voix se trame toujours dans le silence et que le silence a toujours le dernier mot.

2 L'ouvrage dirigé par Claudine Armand est le fruit d'un séminaire régulier organisé sur 3 ans entre 2015 et 2017 à l'université de Lorraine et d'un colloque international portant sur les jeux de voix et de silence dans les arts (juin 2017). L'ensemble des articles rassemblés ici explorent un large panel de médiums qu'il s'agisse de littérature (prose, poésie ou théâtre), de cinéma (courts et longs métrages, documentaires), d'arts visuels (peinture, sculpture, photographie), de spectacle vivant (danse, cirque, etc.) ou de chant lyrique. Cette diversité remarquable d'objets d'étude est une des qualités de ce volume « centré plus particulièrement sur la dialectique voix-silence envisagée comme action et performativité, dans son rapport au corps, lieu de l'émission sonore, de la transmission du souffle et de l'émergence du sens » (25).

3 La préface de David Le Breton, auteur de Éclats de voix: Une Anthropologie des voix (2011), énonce plusieurs axiomes auxquels les différents contributeurs font tour à tour écho: 
«la voix est un artefact» (7); « la voix est la part du corps dans la langue » (8); « corps sans organe, corps subtil, [la voix est] une vibration sonore sur l'infini du silence qui l'enveloppe " (8); «comme le visage, la voix incarne cette infime différence par où chaque individu trouve sa singularité » (8); la voix est « au croisement de l'organique et du sens » (9); « vivre, c'est donner de la voix » (9).

4 La dimension corporelle de la voix est ainsi soulignée dans plusieurs essais et tout d'abord dans celui de Georgia Bruno qui analyse sa pratique du chant lyrique d'un point de vue métaphysique. Soulignant que la voix est «impure » et mâtinée de silence dès son origine (32), elle note que le corps tout entier est engagé dans toute émission vocale et ce, alors même que le chanteur n'existe plus en tant que sujet rationnel et intentionnel (30). Dans l'art du chant, "on se laisse [donc] chanter », on est réceptif plutôt qu'agissant. Dans son évocation d'un spectacle de cirque de Yoann Bourgeois, Nathalie Kloos expose elle aussi que la voix est indissociable du corps. Dans Celui qui tombe, la voix des acrobates ne retentit que transitoirement sur le mode du chant lyrique, en rupture d'une part avec la machinerie bruyante du plateau mobile sur lequel ils évoluent et d'autre part avec les voix enregistrées diffusées par intermittence dans le spectacle. Durant cet intermède vocal, la salle se mue en chambre d'écoute où le spectateur perçoit la fragilité, l'humanité et surtout la corporalité de la voix. On retrouve enfin cette même corporéité dans l'article qu'Ophélie Naessens consacre aux portraits parlants de Gillian Wearing, Justine Pluvinage et Esther Shalev-Gerz mais le lien corps/voix apparaît en creux ou par contrepoint. Renouvelant la pratique documentaire traditionnelle par des jeux frappants de discontinuité et décalages entre corps et voix ou voix et texte et par l'introduction de silences, ces vidéastes explorent en effet la manière dont l'identité se construit en tension entre ce que nous montrons de nous-mêmes et ce que nous gardons enfoui et dérobons au regard des autres. Elles montrent surtout - à l'instar du magistral « Self-Made » réalisé par Wearing en 2010 combien toute voix s'incarne, est littéralement « incorporée ».

Cette voix incarnée, objet du désir et de la jouissance de l'autre, est bien entendu par excellence médiatrice d'une subjectivité. Chloé Larmet explique ainsi comment l'esthétique théâtrale du metteur en scène Claude Régy, fondée sur le "découplement " du geste vocal, conduit le spectateur à entendre l'autre sensible qui parle : " posant des obstacles à la compréhension et au déchiffrement de la voix, il donne à entendre ce qui la sous-tend, à savoir la résonnance silencieuse d'une subjectivité. [...] Ce qui importe ce n'est pas ce que dit cette voix mais ce qu'elle exprime quant à celui qui parle ». (198) La voix, subtil vibrato entre soi, son et sens, signifie (manifeste) donc bien au-delà de ce qu'elle signifie (dit).

6 Elle signifie d'ailleurs parfois d'autant plus qu'elle est travaillée, modulée et modelée pour épouser un sentiment ou mimer une émotion. C'est précisément parce qu'elle avait ce don unique et novateur d'infléchir et de façonner sa voix dans son jeu de scène que l'actrice Vera Komissarjevskaia, interprète de Tchekhov, est devenue l'une des icônes du théâtre russe de la première décennie du $\mathrm{xx}^{\mathrm{e}}$ siècle. Lucie Kempf décrit en ces termes les jeux de voix de Komissarjevskaia au service de l'intensité dramatique: " [Vera] construisait ses rôles d'après des principes musicaux, avec des leitmotiv, des piano, des forte, des silences et un travail sur la tonalité des pièces, jouant tantôt en majeur, tantôt en mineur » (61).

Dans leurs essais consacrés à l'art du roman, Marcin Stawiarski et Anne-Catherine Bascoul montrent, quant à eux, que si donner de la voix, c'est vivre, c'est aussi refuser 
toute soumission : la voix devient vectrice de résistance et d'émancipation. Les trois œuvres choisies par Marcin Stawiarski (un roman de George Eliot, d'Anna Cora Ritchie Mowatt et de James McCourt) figurent des voix féminines défaillantes, muselées ou étouffées; dans chaque cas, la victoire du silence est certes symptomatique d'une oppression et d'un refoulement mais elle dit aussi en creux la puissance transgressive de ces voix féminines et la menace qu'elles représentent potentiellement. Quant au roman Orfeo de Richard Powers étudié par Anne-Catherine Bascoul, il illustre plusieurs formes d'affranchissement: celui du personnage principal à travers la musique et surtout celui du texte qui, musicalisé et polyphonique comme une partition de musique faisant dialoguer voix et silences, s'affranchit de la linéarité et de la bidimensionnalité silencieuse de la page. Dans la poésie de John Agard, poète afro-guyanais auquel Kathie Birat consacre son étude, il est encore question de voix résistante: reprenant les traditions orales de la Caraïbe, Agard fait de sa poésie une performance et utilise le son de sa voix pour faire entendre et dramatiser les silences du passé colonial.

Plusieurs articles accordent enfin une place de choix au silence dont on mesure, à les lire, combien il est " parlant ». On songera tout d'abord au théâtre d'Eugène Ionesco dans lequel, comme le remarque Yannick Hoffert, on ne sait qui du silence ou du bavardage universel représente une plus grande menace : " pour des êtres menacés par le néant, il est vital que la voix s'élève; mais, dans un monde parcouru de multiples bruissements de paroles, la voix n'est souvent [...] que du bruit» (73). On songera encore au théatre de Maeterlinck que Luca Esposito rapproche de l'œuvre picturale de Vilhelm Hammershøi. Si le peintre danois a été qualifié de peintre du silence et les pièces de Maeterlinck interprétées comme une poétique du silence, c'est que dans les deux cas le silence joué ou figuré est « éloquent » (152).

9 Au cinéma, art né « muet » avant de devenir "parlant», le silence joue également un rôle essentiel. Dans un article prégnant intitulé «Du silence au cinéma», José Moure insiste d'ailleurs sur la nécessité de "penser le silence comme un élément structurel qui inquiète le visible, [...] qui en creusant le temps, vidant l'espace et ouvrant des intervalles, met l'image cinématographique en rapport avec un dehors (ou un envers) et par là même la confronte à la part d'absence, de manque, de non-dit, d'irréductible qu'elle recèle » (José Moure, « Du silence au cinéma », 1998, 36-7). Dans les deux longsmétrages de Nuri Bilge Ceylan récompensés à Cannes (" Il était une fois en Anatolie», 2011, et «Winter Sleep », 2014), c'est précisément dans l'alternance de dialogues et de plans silencieux auxquels il confère une densité extrême par le crescendo des voix et un art du cadrage sublimant les paysages d'Anatolie et Cappadoce et les bruits de la nature que le cinéaste turc inquiète le visible, fouille l'intimité sombre de ses personnages et donne à entendre tant de non-dits. Raréfiant la voix, la mettant partiellement hors-jeu, les documentaires de Stéphane Manchematin et Serge Steyer donnent aussi aux sons de la nature et au silence le rôle principal, élaborant une " esthétique et kinésie du corps en écoute » (235). Le silence participe enfin pareillement de l'esthétique de la Nouvelle Vague ou du Nouveau Cinéma allemand à l'instar des films-essais de Godard, Marker et Kluge que Maguelone Loublier prend pour objets de réflexion. Les différentes formes de voix over que l'on rencontre dans ces films ponctuent en effet la bande-image, notamment par leurs silences, introduisant de l'hétérogène, une nouvelle temporalité, révélant l'invisible et donnant accès à l'inouï : «en laissant au silence le temps de se déployer, le montage crée, paradoxalement, une sorte d'arrêt sur image - même si celle-ci reste en mouvement » (175). 
10 Tout autre est le silence que Bridget Sheridan «fait parler» dans son projet photographique sur les chemins de passeurs entre l'Espagne et la France. Mais il n'en est pas moins expressif et sa représentation esthétiquement convaincante. C'est un silence palimpseste, feuilleté du silence des exilés, du silence de la clandestinité et du silence des archives où Sheridan trouve la matière de sa création. C'est avant tout le silence de ses tirages photographiques qui résonnent subtilement mais de manière émouvante de la voix des survivants dont Sheridan superpose le témoignage sur ses images.

11 De façon fort à-propos, les dernières pages de l'ouvrage laissent le dernier mot à des artistes du monde du théâtre et de l'opéra. Qu'il s'agisse de Stephen Langridge, Carmello Agnello, Jean de Pange, Sara Llorca ou Mark SaFranko, tous soulignent l'empiétement et l'entre-tissage créatif et signifiant entre voix et silence(s). On retiendra en particulier avec Sara Llorca que le théâtre est "un territoire perforé » (229) et que le triptyque auteur/texte - acteur - spectateur repose sur du silence et du vide avec lesquels il joue. On notera encore avec Jean de Pange que les textes de théâtre ne sont pas de la littérature mais une " partition orale »; que le silence au théâtre, c'est déjà du théâtre (226-7).

Dans Le Silence dans l'art (2019), Vincent Debiais explique que «dans la pensée médiévale, le silence est établi dans sa relation, positive et négative, au langage et non dans une opposition stricte à la performance de la voix ou à la présence sonore du monde ». Il ajoute que "le silence est une mise en tension de l'état du monde, entre recul, retenue, attente et engagement. (...) il interroge en tant que catégorie anthropologique d'expérience, ce qui ressort à une relation personnelle au langage, à sa privation, à la solitude, à la transcendance ». Le parcours artistique pluridisciplinaire que nous offre l'ouvrage dirigé par Claudine Armand permet de mesurer précisément cela - et que le silence fait écho à la voix et la voix écho au silence...

\section{INDEX}

Mots-clés : voix, silence, corps, souffle, rythme, identité, intimité, arts du spectacle, arts visuels, esthétique

Keywords : voice, silence, body, breath, rhythm, identity, intimacy, performing arts, visual arts, aesthetics

\section{AUTEURS}

\section{CHRISTELLE SERÉE-CHAUSSINAND}

Maître de conférences

Université de Bourgogne

christelle.chaussinand@u-bourgogne.fr 